\title{
Fluid-dynamic control microcatheter used with glue: preliminary experience on its feasibility and safety
}

\author{
Anna Maria lerardi ${ }^{1}$ (D) Elvira Stellato ${ }^{2} \cdot$ Giuseppe Pellegrino $^{2} \cdot$ Cristian Bonelli $^{3} \cdot$ Michaela Cellina $^{4}$. \\ Matteo Renzulli ${ }^{5}$. Pierpaolo Biondetti ${ }^{1}$. Gianpaolo Carrafiello ${ }^{1,6}$
}

Received: 20 May 2021 / Accepted: 21 January 2022 / Published online: 18 February 2022

(c) Italian Society of Medical Radiology 2022

\begin{abstract}
Purpose To evaluate feasibility, safety, and success of peripheral embolization procedures carried out using anti-reflux microcatheter with N-butyl-cyanoacrylate (NBCA) as an embolic agent.

Methods We retrospectively described 11 patients that suffered from active bleeding in different body districts, who underwent embolization procedure using SeQure microcatheter (Guerbet, France) with NBCA glue (Glubran II, GEM Italy) as an embolic agent. The treatments required NBCA volumes ranged from 0.1 to $0.6 \mathrm{~mL}$, with different dilutions with ethiodized oil (Lipiodol, Guerbet, France), depending on the entity of the bleeding. Technical success, clinical success, and complications were evaluated.

Results The procedures were successfully concluded in the totality of the patients, achieving full technical and clinical success. In one patient (9.1\%), a small upstream of embolic material was encountered, without any consequence.

Conclusion This preliminary experience shows that the use of SeQure is feasible and safe with NBCA.
\end{abstract}

Keywords NBCA $\cdot$ Anti-reflux microcatheter $\cdot$ Embolic agents $\cdot$ Bleeding $\cdot$ Embolization

\section{Introduction}

The usage of the anti-reflux microcatheters during embolization procedures has steadily grown during the last decade [1]. Making use of innovative techniques, different catheters prevent upstream of embolization materials, increasing the

Anna Maria Ierardi

amierardi@yahoo.it

1 Radiology Department, Foundation IRCCS Cà Granda Ospedale Maggiore Policlinico, Via Francesco Sforza, 35, 20122 Milan, Italy

2 Postgraduation School in Radiodiagnostics, University of Milan, via Festa del Perdono, 20122 Milan, Italy

3 Healthcare Professional Department, Foundation IRCCS Cà Granda Ospedale Maggiore Policlinico, Milan, Italy

4 Radiology Department, ASST Fatebenefratelli Sacco, piazza Principessa Clotilde 3, 20121 Milan, Italy

5 Department of Radiology, IRCCS Azienda Ospedaliero-Universitaria Di Bologna, Via Albertoni 15, Bologna, Italy

6 Department of Health Sciences, Università Degli Studi Di Milano, Milan, Italy overall effectiveness of the procedures and decreasing the chance of complications.

The use of N-butyl cyanoacrylate (NBCA) has been widely studied and used for interventional radiology purposes. NBCA glue polymerizes into solid form after contact with ions (e.g., water, blood), and it is usually diluted with ethiodized oil which provides radiopacity and increases viscosity [2].

Keys to success include optimizing the ratio of NBCA and ethiodized oil, hence the glue's dilution, delivery of an appropriate volume at a correct speed rate, and assessment of the embolic effect with imaging. All these factors must be carefully evaluated to avoid severe complications. For instance, early polymerization of glue can occur inside the microcatheter, leading to the occlusion of the lumen and the potential loss of access, making the procedure harder and associated with a higher complications chance [3].

The most dangerous complication of this procedure is non-target embolization: unintentional reflux of embolic agent that can upstream to unwanted vessels, embolize them, and cause severe conditions for the patient [4]. SeQure (Guerbet, France) is a microcatheter recently introduced for peripheral embolization procedures; 
it works using flow dynamics to create a fluid barrier designed to deliver embolic agents to the target and reduce the risk of non-target [5]. SeQure has already been successfully used with particles as embolic agents in other procedures in the literature [6].

No data are available about the use of SeQure with NBCA and much less about the effectiveness of antireflux system with glucose solution and glue. The aim of our study was to evaluate feasibility, safety, and success of peripheral embolization procedures carried out using anti-reflux microcatheter with NBCA as embolic agent.

\section{Materials and methods}

The study was held as a single-center retrospective study. Our Ethics Committee approved the study. This case series includes patients who underwent an embolization procedure using SeQure microcatheter (Guerbet, France) with NBCA glue (Glubran II, GEM Italy) as embolic agent, in the emergency setting. In the study period, about 6 months, a total of 11 patients ( 6 men and 5 women) with a mean age of 79.3 years (range $50-91$ years old) were treated. The examined patients suffering from active bleeding in the following districts: psoas (Fig. 1) pectoralis, obturator, intercostal (Fig. 2) and rectus abdominis muscles, renal tumors (angiomyolipoma) (Fig. 3). All patients signed informed consent.

Characteristics and bleeding causes are shown in Table 1.
Fig. 1 Retroperitoneal bleeding in COVID-19 patient (patient 8 of Table 1): Contrast-enhanced CT shows active bleeding (white arrow); angiogram of ileolumbar artery $(\mathrm{C}, \mathrm{D})$ and embolization with glue (1:2) (E, F)
Fig. 2 Hemothorax with active bleeding at contrast enhanced CT (A, white arrow) (patient 11 of Table 1); selective embolization of intercostal arteries with SeQure microcatheter $(B, C)$ and complete embolization with glue (1:2) (D)
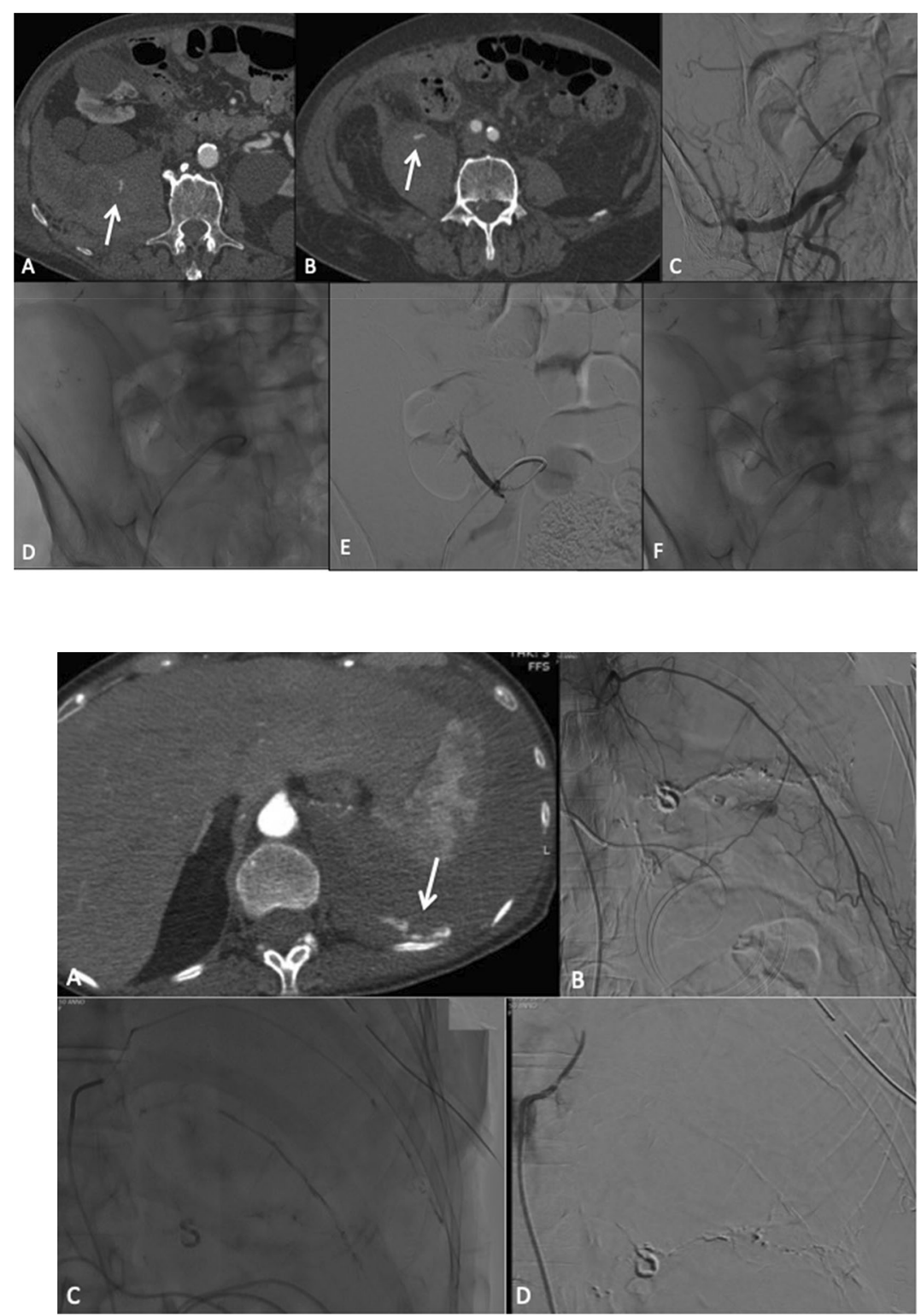
Fig. 3 Selective angiogram of right renal artery shows angiomyolipoma (A) (patient 4 of Table 1); angiogram carried out during the procedure (B); fluoroscopic images show a good distribution of the glue $(1: 2)(\mathrm{C}, \mathrm{D})$; final angiogram demonstrates complete embolization of the angiomyolipoma and complete preservation of renal vascularization $(\mathrm{E})$

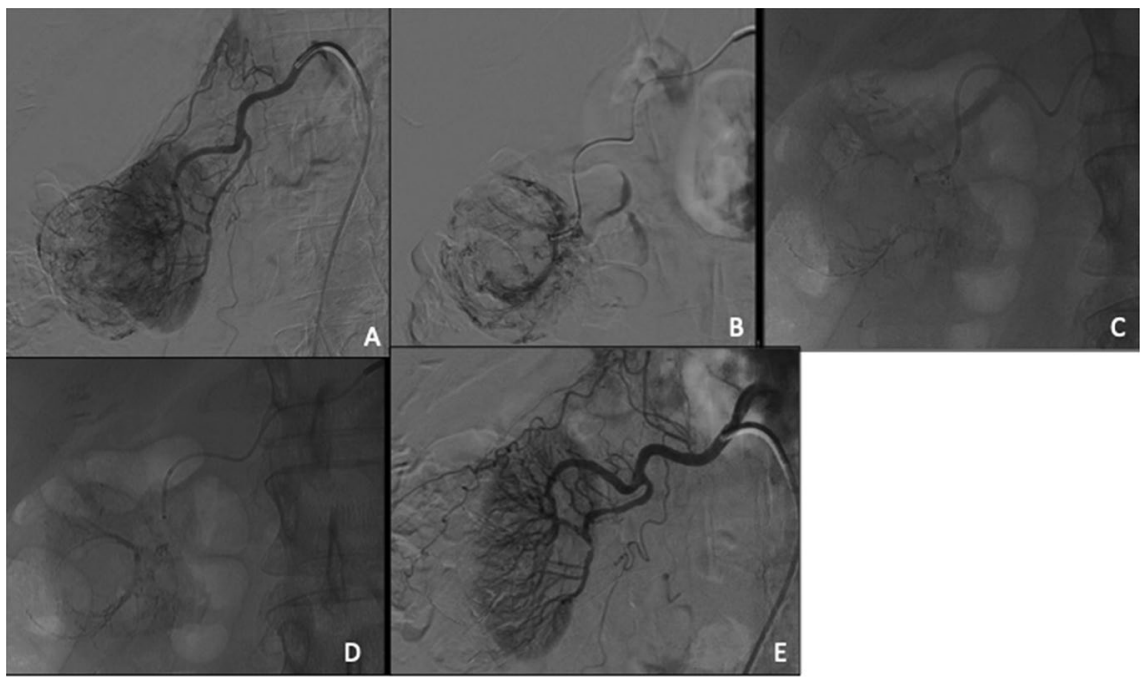

Table 1 Patients' and treated bleedings' characteristics

\begin{tabular}{llll}
\hline Patients & Age and sex & Bleeding cause & Bleeding site \\
\hline 1 & $74-$ Male & Anticoagulated-COVID-19+ & Inferior epigastric artery \\
2 & $65-$ Female & Anticoagulated-COVID-19+ & Pectoralis muscle \\
3 & $84-$ Male & Anticoagulated-COVID-19+ & Psoas muscle (3 lumbar arteries) \\
4 & $88-$ Female & Trauma & Renal angiomyolipoma \\
5 & $85-$ Male & Anticoagulated & Psoas muscle (2 lumbar artery and ileolumbar artery) \\
6 & $78-$ Male & Trauma & Renal artery \\
7 & $77-$ Male & Anticoagulated & Psoas muscle (2 lumbar artery and ileolumbar artery) \\
8 & $81-$ Female & Anticoagulated & Psoas muscle (1 lumbar artery and ileolumbar artery) \\
9 & $70-$ Male & Trauma-Anticoagulated & Obturator muscle \\
10 & $91-$ Female & Anticoagulated & Inferior epigastric artery \\
11 & $50-$ Female & Iatrogenic - Drainage Deployment & 3 Intercostal arteries \\
\hline
\end{tabular}

After local anesthesia (lidocaine 2\%), we inserted a $5 \mathrm{Fr}$ sheath in the femoral artery. Through a 5Fr diagnostic catheter on a guidewire, catheterization of the selected artery was executed. After that, a selective angiogram was performed and found an arterial blush in all patients, except that in patient with renal angiomyolipoma. Once evaluated the entity of the bleeding, a 2.7 F SeQure anti-reflux microcatheter was advanced on a guidewire (Fathom 16, Boston Scientific, Natick, MA) in ten patients out of eleven; a 2.8 F catheter was used on the other patient. The size of the catheter was chosen based on the caliber of the vessel to embolize, and in all cases, it was advanced distally up the bleeding. The next step of the procedure involved the preparation of the glue and its dilution according to the clinical need. A dilution of 1:2 glue to ethiodized oil (Lipiodol, Guerbet, France) was chosen for $91 \%$ of the treated patients. The only exception was made for patient 5 , where a small volume of glue was required $(0,1 \mathrm{~mL})$, opting for a 1:1 dilution. The volume used, ranging between 0.1 and $0.6 \mathrm{~mL}$, depends on the entity of the bleeding and the caliber of the artery to embolize. The dilution was chosen on the basis of the desired grade of polymerization.

The outcome of the procedures was evaluated considering three different parameters:

- TS (technical success) was defined as the possibility to arise the bleeding vessel and carry out a target embolization.

- CS (clinical success) was defined as the cessation of the bleeding in the $24 \mathrm{~h}$ following the procedure

- Safety was defined as the evaluation of onset of immediate or delayed complications, recorded on the basis of SIR classification [7]. 


\section{Results}

$72.7 \%$ of treated bleedings were registered in anticoagulated patients (two of them diagnosed with a COVID-19 infection), $18.2 \%$ after trauma, and the remnant $9.1 \%$ had an iatrogenic cause.

The technical success was achieved in the totality of the interventions, since SeQure microcatheter was effectively led to the desired site and embolization was performed. Clinical success was achieved in $100 \%$ of patients, since in all cases, embolization was effectively performed using the combination of SeQure microcatheter and glue. No harmful or life-threatening complications were observed during the procedures. In one patient $(9.1 \%)$, we encountered the onset of slight embolic agent reflux that did not cause any type of consequence for the patient.

\section{Discussion}

The usage of an anti-reflux catheter, as shown, has a major advantage over different catheters in the form of being less likely to cause a non-target embolization [1, $5,6]$. Innovative techniques have made each anti-reflux microcatheter suitable for different procedures [5]. Transarterial chemoembolization (TACE) of liver cancer has shown good results when performed with a dual-balloon infusion microcatheter [8] that allowed the isolation of the treated area while still guaranteeing the blood flow through a bypass.

SeQure, the microcatheter we used for all the procedures of the series, has two unique side slits near the main tip which allows the fluid to exit in parallel with the course of the catheter, creating a protective fluid barrier at the sides of the catheter that prevents from unwanted upstream of embolizing material. SeQure has been successfully used combined with microspheres in TACE procedures [9] and for the treatment of severe hemoptysis in bronchiectasis patients [6] showing a virtually full protection from reflux.

No other articles in the literature have talked about the combined usage of SeQure and NBCA in vitro or in vivo scenarios, so there has been no evidence of its efficacy and safety so far. A partial reflux of glue was experienced in patient number 10 , while the procedure was in its final phase. The most likely hypothesis is that being the target almost fully embolized at the end of the operation, a small amount of excess glue found its way upstream. This hypothesis needs to be confirmed by further experimentation and future studies on the topic.

Our study presents some limitations: firstly, its retrospective style; then, the patient cohort was small, and hence, prospective randomized clinical studies are required to define the clinical benefits of the association of the devices reported compared with the use of a conventional microcatheter in terms of safety and patient outcomes.

Therefore, to date, we are not able to explain the action of the diluted glue conveyed by glucose solution, as usually recommended by guidelines, neither we can be sure that glucose solution contributes to the anti-reflux mechanism flowing out of the side slits. [3].

In conclusion, this preliminary experience showed us that SeQure is a very efficient catheter that demonstrated its safety with NBCA. The effectiveness of glue in peripheral embolization is well known; the possibility to use it safely with SeQure represents a new application of this new microcatheter. It should be considered a promising field of application by experienced operators.

Of course, this combination needs to be studied more deeply to understand optimal dilutions of glue with SeQure, whether it can be the gold standard for some indications, which are the best indications for each treatable conditions and if there are cases where it is better to opt for different approaches.

Based on this preliminary experience, bleeding embolization with glue using Sequre microcatheter may be considered feasible and safe.

Authors' contributions AMI and GC contributed to conceptualization; A.M.I was involved in methodology and resources; PB contributed to software and supervision; CB, ES were involved in validation; GP contributed to formal analysis, writing - original draft preparation, visualization; G.C. was involved in investigation, project administration; E.S. contributed to data curation; A.M.I.., MC; MR were involved in writing-review and editing.All authors have read and approved the final manuscript.

Funding This research received no external funding.

\section{Declarations}

Conflict of interest The authors declare no conflict of interest and have not any financial relationship with any sponsoring organization.

Availability of data Data are available in the "References" section for each cited material.

Ethics approval All procedures performed in studies involving human participants were in accordance with the ethical standards of the institutional and/or national research committee and with the 1964 Helsinki declaration and its later amendments or comparable ethical standards. Institutional Review Board (IRB) approval was obtained for this retrospective study.

Consent to participate Informed consent was obtained from all subjects involved in the study. 
Consent to publication Consent for publication was obtained for every individual person's data included in the study.

\section{References}

1. van Roekel C, van den Hoven AF, Bastiaannet R, Bruijnen RCG, Braat AJAT, de Keizer B, Lam MGEH, Smits MLJ (2021) Use of an anti-reflux catheter to improve tumor targeting for holmium-166 radioembolization-a prospective, within-patient randomized study. Eur J Nucl Med Mol Imaging 48(5):1658-1668. https://doi.org/10.1007/s00259-020-05079-0

2. Hill H, Chick JFB, Hage A, Srinivasa RN (2018) N-butyl cyanoacrylate embolotherapy: techniques, complications, and management. Diagn Interv Radiol 24(2):98-103. https://doi.org/ 10.5152/dir.2018.17432

3. Takeuchi Y, Morishita H, Sato Y, Hamaguchi S, Sakamoto N, Tokue H, Yonemitsu T, Murakami K, Fujiwara H, Sofue K, Abe T, Higashihara H, Nakajima Y, Sato M. Committee of Practice Guidelines of the Japanese Society of Interventional Radiology. Guidelines for the use of NBCA in vascular embolization devised by the Committee of Practice Guidelines of the Japanese Society of Interventional Radiology (CGJSIR), 2012 edition. Jpn J Radiol 2014;32(8):500-17. doi: https://doi.org/10.1007/ s11604-014-0328-7.

4. López-Benítez R, Richter GM, Kauczor HU, Stampfl S, Kladeck J, Radeleff BA, Neukamm M, Hallscheidt PJ (2009) Analysis of nontarget embolization mechanisms during embolization and chemoembolization procedures. Cardiovasc Intervent Radiol 32(4):615-622. https://doi.org/10.1007/s00270-009-9568-9

5. Pardo Moreno P, Ruiz Villaverde G, Ciampi Dopazo JJ, Navarro VP (2020) Novel reflux-control microcatheter: an alternative to maximize the effect of superselective embolization. Intervencionismo 20(3):126-133. https://doi.org/10.30454/2530-1209. 2020.3.4

6. Angileri SA, Rodà GM, Arrichiello A, Signorelli G, Di Meglio L, Gurgitano M, Di Bartolomeo F, Ierardi AM, Paolucci A, Carrafiello G (2020) Efficacy, safety and usability of bronchial artery embolization using a new anti-reflux microcatheter in the management of haemoptysis. Acta Biomed 91(10-S):e2020009. doi: https://doi.org/10.23750/abm.v91i10-S.10265.

7. Khalilzadeh O, Baerlocher MO, Shyn PB, Connolly BL, Devane AM, Morris CS, Cohen AM, Midia M, Thornton RH, Gross K, Caplin DM, Aeron G, Misra S, Patel NH, Walker TG, Martinez-Salazar G, Silberzweig JE, Nikolic B (2017) Proposal of a new adverse event classification by the society of interventional radiology standards of practice committee. J Vasc Interv Radiol 28(10):1432-1437

8. Monsky WL, Padia SA, Hardy AH (2017) Dual-balloon infusion microcatheter for selective drug-eluting bead transarterial chemoembolization: initial feasibility study. Diagn Interv Radiol 23(6):454-460

9. van den Hoven AF, Lam MG, Jernigan S, van den Bosch MA, Buckner GD (2015) Innovation in catheter design for intra-arterial liver cancer treatments results in favorable particle-fluid dynamics. J Exp Clin Cancer Res 34(1):74. https://doi.org/10.1186/ s13046-015-0188-8

Publisher's Note Springer Nature remains neutral with regard to jurisdictional claims in published maps and institutional affiliations. 